\title{
Metabolic rate links density to demography in Tetrahymena pyriformis
}

\author{
John P DeLong and David T Hanson \\ Department of Biology, University of New Mexico, Albuquerque, NM, USA
}

\begin{abstract}
Many populations show density-dependent growth rates. We suggest that population growth rate may be connected to density through the density dependence of metabolic rate. If metabolic rate is an index of the total biochemical work being done by an organism, then as populations grow and density suppresses metabolic rate, the rate of reproduction should slow and the ability to avoid death should diminish. To test this idea, we grew axenic populations of a single-celled protist, Tetrahymena pyriformis, in laboratory microcosms, and measured metabolic rate and density. We also estimated division (birth) and death rates. The proposed connection was supported by two observations. First, increasing population density suppressed per-capita metabolic rate in accordance with the predictions of a resource-division model. The same pattern was shown with experimentally altered densities. Second, per-capita metabolic rate was positively related to percapita division rate and negatively related to per-capita death rate. Thus, the particular pattern of population growth and regulation depends on exactly how individuals respond energetically to the density of conspecifics, and how they allocate metabolism to maintenance and production. The physiological basis of population regulation in this system is based on the constraints imposed on the total metabolic work that individual cells can perform.

The ISME Journal (2009) 3, 1396-1401; doi:10.1038/ismej.2009.81; published online 2 July 2009

Subject Category: microbial population and community ecology

Keywords: Tetrahymena; population regulation; population energetics; density dependence; metabolic ecology; metabolic rate
\end{abstract}

\section{Introduction}

A common observation in ecology is that population size does not increase indefinitely. As a population grows, a feedback loop is generated that reduces the per-capita growth rate (PGR) of the population, resulting in density-dependent population regulation (Pearl, 1927; Turchin, 1999; Berryman et al., 2002; Sibly et al., 2005). The mechanism of regulation operates at the individual level; densitydependent regulation occurs when increasing population size increases mortality, decreases natality or alters migration patterns of individuals in the population (Hixon and Carr, 1997; Turchin, 1999; van Aarde et al., 1999; Ohman and Hirche, 2001; Lambers et al., 2002).

Conventionally, density dependence has been studied by linking density to population parameters (for example, population-level birth rate, death rate) or by linking density to PGR in time-series data (Bulmer, 1975; Woiwod and Hanski, 1992; Turchin,

Correspondence: JP DeLong, Department of Biology, University of New Mexico, Albuquerque, NM 87131, USA.

E-mail: john.delong@yale.edu

Received 21 April 2009; revised 2 June 2009; accepted 3 June 2009; published online 2 July 2009
1999; Sibly et al., 2005). However, demographic parameters may not always respond directly to density. They may in some cases respond to factors affected by density, such as food availability or predation risk. We suggest that, in the case of regulation through resource availability, some type of physiological state acts as an intermediary between density and demographic parameters, and therefore PGR. More precisely, density, through intraspecific competition for resources, may affect the physiological state of the organism, and the organism may employ alternative strategies to maximize fitness, such as when and whether to reproduce or migrate. In this context, a salient feature of physiological state is metabolic rate, the rate of acquisition, processing and allocation of material resources (Brown et al., 2004). Although other physiological measures may be relevant, we argue that metabolic rate should be a good physiological intermediate because it indexes the amount of work being done by an organism at the time of measurement. Furthermore, as metabolic rate is also influenced by temperature and body size (Gillooly et al., 2001), positing metabolic rate as an intermediary will allow us to investigate how density interacts with other environmental variables to produce specific demographic patterns. 
For many types of organisms, metabolic rate can vary considerably within an individual or species depending on activity level and resource availability (for example, Secor and Diamond, 1998; Schmoker and Hernandez-Leon, 2003). Therefore, metabolic rate is plastic enough to act as a state variable. From the point of view of population regulation, variation in resource supply should lead to altered metabolic rates and changes in the allocation of energy and resources to growth, reproduction or survival, driving changes in population growth rate (Tonn et al., 1994; Lewis et al., 2001; Schmoker and Hernandez-Leon, 2003; Forrester et al., 2006). As metabolism fuels production (Brown and Sibly, 2006), a reduction in metabolic rate should decrease the rate at which offspring can be produced. Similarly, survival should be enhanced when metabolism can be allocated to cell maintenance or other defense mechanisms, such as avoidance of predation. As the total metabolic rate declines, reproduction or survival must decline as well.

DeLong and Hanson (2009) developed a simple resource-division model for density-dependent metabolism. Given a fixed rate of supply of a limiting resource to a population $\left(R_{\mathrm{tot}}\right)$, the average per-capita availability of resources $\left(\bar{R}_{\mathrm{pc}}\right)$ will be $R_{\mathrm{tot}}$ divided by the density of individuals $(N)$. If these resources are metabolism supporting, then the per-capita metabolic rate $\left(\bar{B}_{\mathrm{pc}}\right)$ will be related to $\bar{R}_{\mathrm{pc}}$ by:

$$
\bar{B}_{\mathrm{pc}}=\frac{c R_{\mathrm{tot}}}{N^{\alpha}}
$$

where $C$ is a conversion factor that translates how a unit of resource yields a unit of metabolism. The effect of adding additional individuals may be modified by the level of intraspecific competition, $\alpha$, such that deviations from a perfect inverse distribution of resources may occur. Equation (1) predicts density-dependent $\bar{B}_{\mathrm{pc}}$ in a form that is influenced by resource availability and the level of intraspecific competition, but clearly there is a maximum metabolic rate that bounds this function at lower densities. The model provides us with a direct link between density and the physiological state of the average individual. The connection between $\bar{B}_{\mathrm{pc}}$ and demographic rates is that $\bar{B}_{\mathrm{pc}}$ reflects the amount of biochemical work being done by an organism, as each unit of metabolism is related to the production of adenosine triphosphate. A density-induced constraint on $\bar{B}_{\mathrm{pc}}$ is a constraint on the total work being done by an individual organism, which should translate into effects on growth, survival and movement. We can therefore view birth $(b)$, death $(d)$ and migration $(m)$ rates as functions of $\bar{B}_{\mathrm{pc}}$, which given its connection to density, provides an explicit energetic link between density and PGR.

Population regulation of single-celled eukaryotes (protists) has been studied extensively. Protists are particularly well suited for testing our hypothesis that the metabolic rate could serve as a physiological link between density and demographic parameters for three reasons. First, many experimental populations show sigmoidal growth, which implies densitydependent PGR (for example, Gause, 1934). Second, individual metabolic rates of protists are highly plastic and are negatively related to density (for example, Pace and Kimura, 1944; Nässberger and Monti, 1984, DeLong and Hanson, 2009). Third, axenic cultures can be grown and the population change, total population metabolic rate and demographic parameters can be monitored simultaneously.

In this study, we created microcosm populations of the ciliate Tetrahymena pyriformis, and let population size increase to carrying capacity. We measured whole-population metabolic rate $\left(B_{\text {pop }}\right.$ as oxygen consumption), population size, division (birth) and death rate, and cell size as the population grew, and we used these data to determine how $\bar{B}_{\mathrm{pc}}$ is related to cell density and demographic parameters. Microcosm populations were closed, so migration was not possible, and we do not address this process further. Specifically, we predicted that $\bar{B}_{\mathrm{pc}}$ would vary with the inverse of $N$ raised to some power, $\alpha$, and that birth rates would vary positively, and death rates, negatively, with to $\bar{B}_{\mathrm{pc}}$. Our results show that recognizing the metabolic link between density and demography can greatly improve our understanding of the physiological basis of population regulation.

\section{Materials and methods}

\section{Microcosms}

We grew four axenic populations of T. pyriformis to resource carrying capacity in $10-\mathrm{ml}$ microcosms in 16-ml vials sealed with septum tops. We maintained a regular resource supply rate by removing $0.7 \mathrm{ml}$ of well-mixed culture daily and replacing it with $0.7 \mathrm{ml}$ fresh, sterile food medium (Tetrahymena medium from Carolina Biological Supply (Burlington, NC, USA) reduced in concentration 1:3 medium:sterile water). We maintained aerobic conditions by aerating microcosms with filtered air (using sterile $0.2 \mu \mathrm{m}$ syringe filters in a vial-length needle pushed through the septum). This prevented cells from switching to anaerobic metabolism and allowed the use of oxygen consumption as a measure of metabolic rate. A second bent needle vented the headspace and magnetic stir-bars mixed the microcosms. We conducted all extractions and additions to the microcosms under a laminar flow hood using sterile techniques. To reduce the potential for contamination, we added penicillin (10000 IU) and streptomycin $\left(10000 \mu \mathrm{g} \mathrm{ml}^{-1}\right)$ at a 1:100 dilution with the food medium. Bacterial contamination also can be detected by a cloudy appearance to the cultures. All data reported here are from microcosms confirmed to be sterile by plate streaking (1-2 drops sample per culture for 1-2 days at $37^{\circ} \mathrm{C}$ ) at the beginning and end of the experiment, and by the absence of a cloudy appearance. There- 
fore, the rate of oxygen consumption measures only the metabolic rate of $T$. pyriformis.

\section{Respiration}

We used a fluorescent oxygen needle probe (FOXY18G with overcoat, Ocean Optics Inc., Dunedin, FL, USA) to measure the change in molar fraction of dissolved oxygen because of metabolic activity (see DeLong and Hanson, 2009 for more details). We maintained microcosms at $19-21^{\circ} \mathrm{C}$ using a water bath and corrected oxygen consumption values to $20^{\circ} \mathrm{C}$. For example, we converted a rate measured at $19.5^{\circ} \mathrm{C}$ to the equivalent rate at $20^{\circ} \mathrm{C}$ on the basis of the exponential dependence of metabolic rate on temperature (Gillooly et al., 2001):

$$
\text { Conversion factor (19.5 to } 20)=\frac{e\left(-E / k T_{20}\right)}{e\left(-E / k T_{19.5}\right)}
$$

where $E$ is the activation energy set at $0.61 \mathrm{eV}$ (from Gillooly et al., 2001), $T$ is temperature (K) and $k$ is the Boltzmann's constant (eV per K). Average percapita metabolic rate was given by dividing the whole-population oxygen consumption rate by cell density (see below).

To ensure that long-term changes in physiology or evolution were not the cause of the density dependence of metabolic rate, we measured the oxygen consumption in three samples with manipulated densities. These samples came from one culture at the end of the experiment. Densities were altered to (i) approximately carrying capacity, (ii) about two times carrying capacity and (iii) to approximately one-third of carrying capacity (solid circles, Figure 2). To do this, we lightly centrifuged samples (1-2000 r.p.m. for $\sim 1 \mathrm{~min}$ ) and collected cells either from the bottom of the tube (giving a higher density) or from the top of the tube (giving a lower density). There was no indication of any damage to cells or altered behavior. We immediately measured oxygen consumption of these samples in the same way as we did with the time-series experiment.

\section{Density and cell division rates}

We sub-sampled $(0.1 \mathrm{ml})$ the oxygen consumption samples and counted the number of individuals live under a stereo microscope. We measured sample volumes as mass with an electronic balance, and diluted them if necessary with the resource medium until cell densities were low enough to count accurately. We also estimated birth rate by counting the number of actively dividing cells while measuring cell density (Cameron and Jeter 1970). Cells whose division was far enough along in mitosis to appear as a 'figure 8' rather than a pear-shaped cell were counted as dividing, and the per-capita birth index was simply the proportion of cells actively dividing. As the birth index does not have an $a$ priori specified time component (that is, it is not a rate), we adjusted the time frame of the birth index to match the population growth rate at very low densities, when the death rate is presumably negligible, to get the birth rate $(b)$. To achieve this, we divided the birth index by 18.4 (units are $\mathrm{t}^{-1}$ ).

\section{Death rates}

We calculated per-capita death rates $(d)$ by subtracting $b$ from the per-capita change in population size $(\Delta N)$. We did this in several steps. First, we fitted a continuous logistic growth model to our time-series data for $N$ (all data together) using non-linear leastsquare regression (see Figure 1). Other growth models may have worked just as well; our purpose was to create a simple approximation of the shape of population growth which the logistic model provided. Of course, the choice of model would influence the shape of the death rate function, but would not change our general results. With this approximation of the population growth, we calculated $\Delta N$ for 1-min interval through the time frame of the study. Second, we estimated the relationship between $b$ and time $\left(b=0.0077 e^{(-0.00003 \mathrm{~m})} ; R^{2}=0.31\right)$, again using nonlinear least-square regression. Third, as we then knew both $\Delta N$ and $b$ for each 1-min interval, we could calculate $d$ for each minute $(t)$ using:

$$
\mathrm{d}(t)=b(t)-\frac{\Delta N(t)}{N(t)}
$$

\section{Cell size}

We estimated cell size as cell volume, assuming neutral buoyancy of cells. Throughout the course of the experiment, we photographed a sample of live cells under a microscope and measured the outer

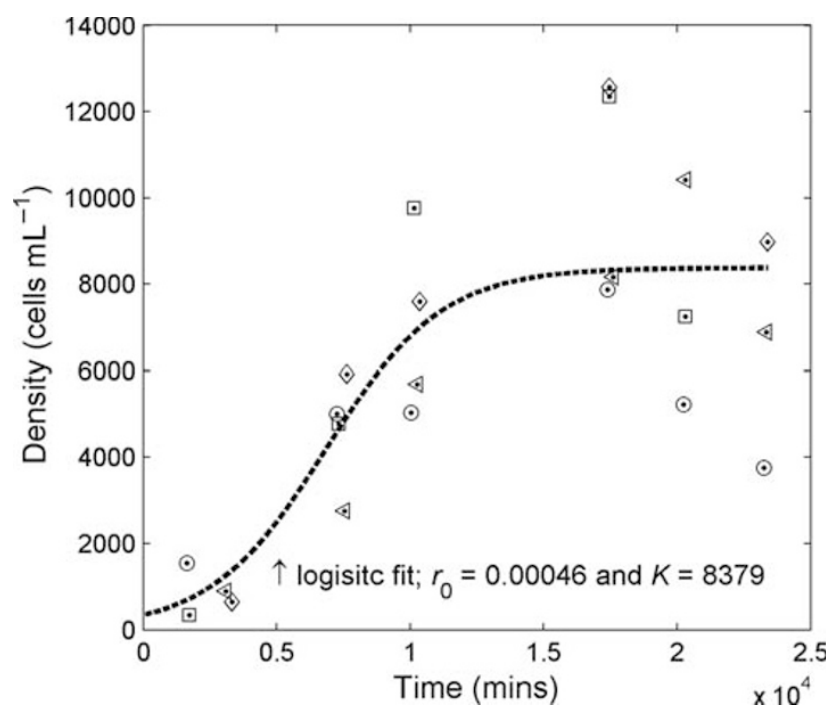

Figure 1 Growth trajectories of four microcosm populations of Tetrahymena pyriformis (different symbols represent different replicate microcosms). The dashed line represents the trajectory as fitted by a logistic growth model with all replicates analyzed together. The fit of the model was used to link population growth to death rates through 1-min intervals through the time series. 
dimensions of 6-61 cells per sample (more than 40 cells in most cases) along two axes. Then, we calculated cell volume using the formula for a prolate spheroid.

\section{Results}

The four replicate populations grew in sigmoidal manner and reached an approximate carrying capacity, $K$, in 12-16 days (Figure 1). There was considerable between-replicate variation in trajectory and $K$ despite efforts to standardize microcosm setup and resource provisioning rate. This may have resulted from the pulsed nature of the resource addition or asynchrony caused by variation in the exact time at which populations were sampled. Parameters for the logistic model were $r_{\max }=0.00046 \mathrm{~min}^{-1} \quad(95 \%$ CI: $-0.00034-0.00059)$ and $K=8379$ (95\% CI: 6942-9816).

As predicted by Eq. 1, there was an inverse relation between $\bar{B}_{\mathrm{pc}}$ and population density $N$ (Figure 2). As $N$ increased, there was a rapid but decelerating reduction in $\bar{B}_{\mathrm{pc}}$. A fit of Eq. 1 to this data using non-linear least-square regression was very tight $\left(R^{2}=0.97\right)$ and yielded parameter estimates of $\alpha=0.84$ (95\% CI: 0.77-0.91) and constant $C_{R}=0.26$ (95\% CI: 0.14-0.38), which here represents the product of $R_{\text {tot }}$ and $c$ in Equation (1). Thus, the first part of the link that metabolic rate is related to

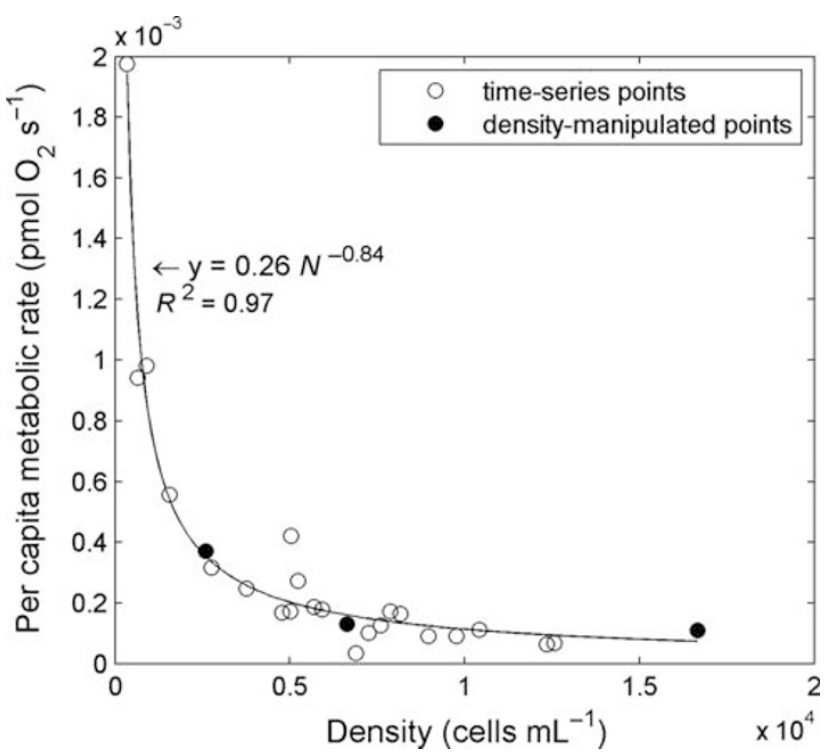

Figure 2 The relationship between density and per-capita metabolic rate $\left(\bar{B}_{\mathrm{pc}}\right)$ for four replicated population growth series (open circles) of Tetrahymena pyriformis. This figure shows that density has a negative effect on per-capita metabolic rate that follows very closely the pattern predicted by Equation (1), which was fit to the data using non-linear least-square regression. Rapid augmentation and reduction of density measurements follow the curve very closely (solid circles), indicating that the suppression of metabolic rate as a result of reduced resource availability was nearly instantaneous and not the result of long-term adjustments of cells to their environment. density in an inverse manner according to Eq. 1 is supported. The second part of the link that demographic rates are related to metabolic rate was also supported. Per-capita birth rates $(b)$ were positively and death rates $(d)$ were negatively related to $\bar{B}_{\mathrm{pc}}$, as predicted (Figure 3). Birth and death rates therefore were linked to density through the metabolic rate of individuals.

The density-altered samples showed the same relation between $\bar{B}_{\mathrm{pc}}$ and density as that of the samples in which density was changed by population growth. Therefore, the negative feedback of density on $\bar{B}_{\text {pc }}$ was not due to a long-term adjustment of individuals to their environment, physiological changes related to stationary phase or to changes associated with turnover of individuals in the population. Rather, it was a nearly an instantaneous drop in biochemical work caused by a decline in the per-capita availability of resources, which itself was caused by rising density.

As body mass has a strong effect on $\bar{B}_{\mathrm{pc}}$ across species (Fenchel and Finlay, 1983), we checked to see whether the decrease in metabolic rate was due to declining cell size. Volume decreased nonsignificantly $(P>0.25)$ throughout the course of the experiment (Figure 4a). Much of this apparent decrease seems to be due to a particularly large cell volume estimate for one replicate early in the experiment that was also the smallest sample. Mass-corrected $\bar{B}_{\mathrm{pc}}\left(\bar{B}_{\mathrm{pc}}{ }^{*}\right.$ mass $\left.^{-0.75}\right)$ showed the same inverse relationship with density as wholeorganism metabolic rate, but with $\alpha=0.79$

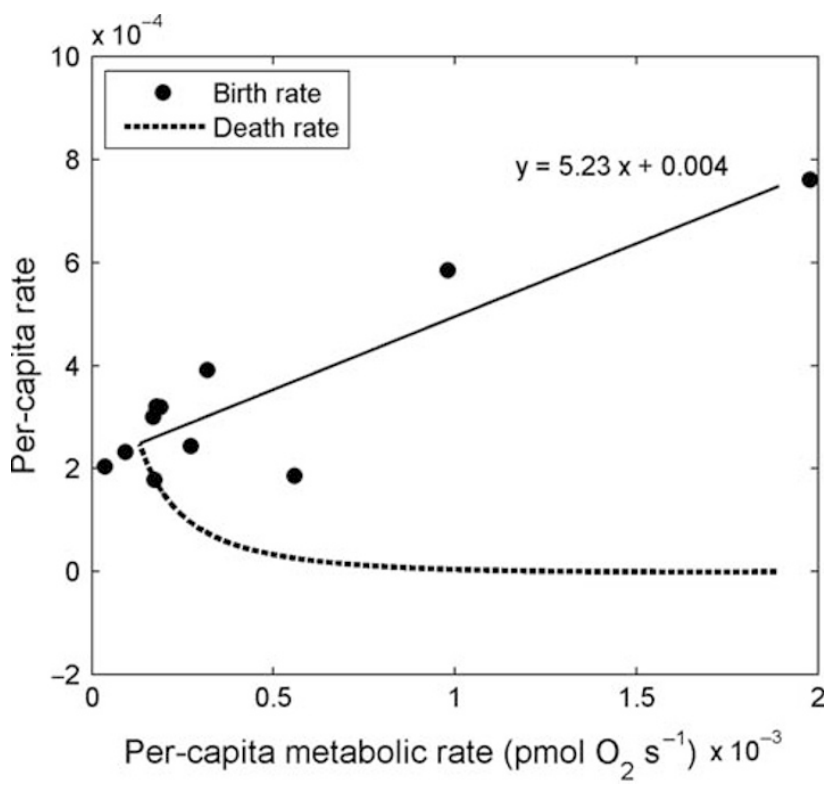

Figure 3 The relationship between birth and death rates and percapita metabolic rate $\left(\bar{B}_{\mathrm{pc}}\right)$ for Tetrahymena pyriformis. The raw per-capita birth rate data are plotted as solid circles, and an empirical fit of the relationship between $\bar{B}_{\mathrm{pc}}$ and per-capita birth rate is shown as a solid line. The death rate is shown as a dashed line and was calculated using Equation (2) and was linked to $\bar{B}_{\mathrm{pc}}$ through 1-min time intervals (see text). 
1400
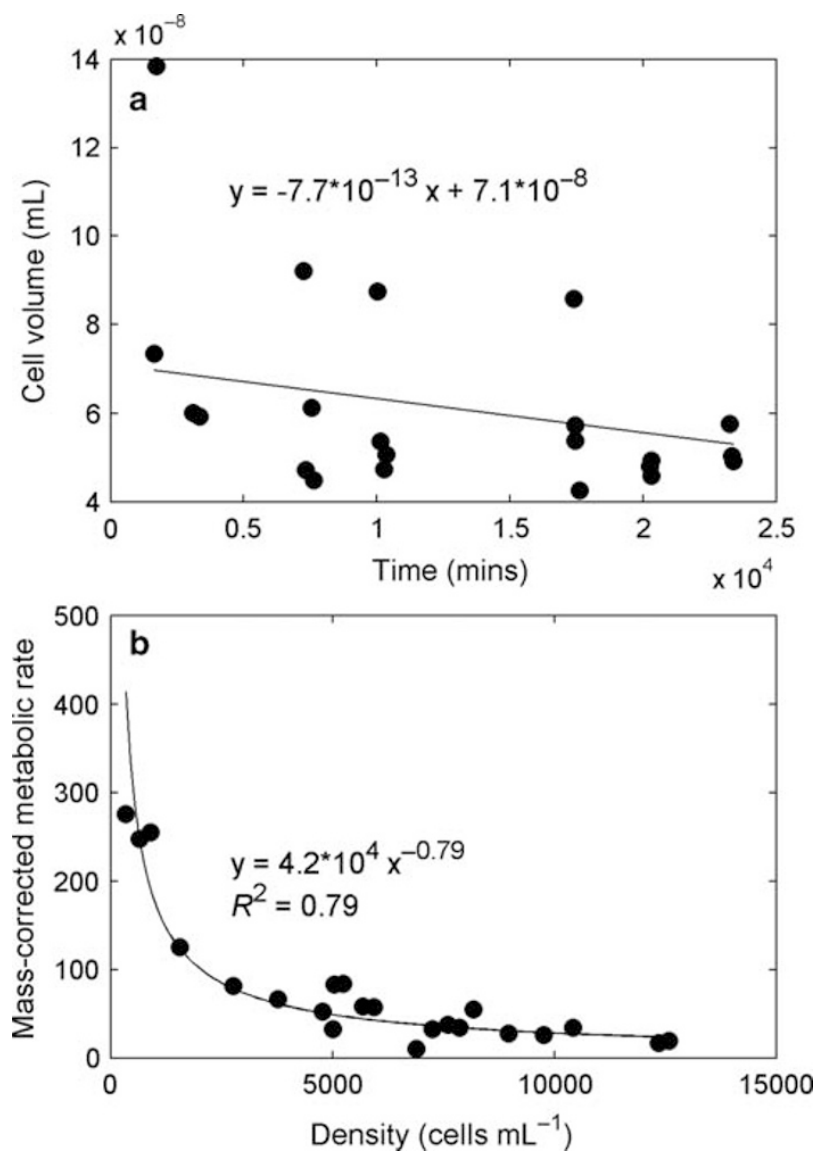

Figure 4 Average Tetrahymena pyriformis cell mass decreased slightly through time (a), without changing the density dependence of per-capita metabolic rate $\left(\bar{B}_{\mathrm{pc}}\right)(\mathbf{b})$, here corrected for cell volume.

(Figure 4b), indicating that body mass changes were not driving either the reduction in metabolic rate or the effects on division and death rates.

\section{Discussion}

Density-dependent population regulation seems to be a widespread phenomenon (Sibly et al., 2005). It is a straightforward consequence of competition for increasingly scarce resources as population size increases. Yet, the changes in birth, death or migration rates that in combination result in a lower PGR must reflect changes in the acquisition and allocation of resources by individuals in response to varying resource supply. Our results show that the metabolic rate of individual $T$. pyriformis is suppressed with increasing density, and this suppression is linked to reduced birth rates and elevated death rates. The first link that density suppresses $\bar{B}_{\mathrm{pc}}$ through its effect on resource availability, is well supported by this study as well as our earlier experimental work (DeLong and Hanson, 2009). That suppression operated across a wide range of densities, not just near carrying capacity. The second link, between metabolic rate and demographic para- meters, is supported by the relationships found here, although experimental confirmation of the link would be welcome. Overall, we find support for the idea that the decline in metabolic rate associated with increased density can be seen as a decline in the ability of the organism to conduct metabolic work that allows reproduction or cell maintenance, leading to density-dependent PGR.

We observed a 20-fold variation in $\bar{B}_{\mathrm{pc}}$. This metabolic suppression likely is related to intraspecific competition for metabolism-supporting resources. That the data are well predicted by our resource-distribution model strongly implicates this interpretation. In addition, a series of studies with protists showed that starvation can reduce $\bar{B}_{\mathrm{pc}}$ up to about an order of magnitude, indicating that access to food resources has a direct and rapid effect on the energy use of these organisms (Fenchel and Finlay, 1983). Unlike earlier studies that measured starvation-state respiration rates by transferring individuals into a non-nutrient media, we measured metabolic rates of an active population growing in response to a fixed rate of food provisioning. Thus, the metabolic rate suppression we observed in this study reflects a reduction of resource availability associated with intraspecific competition, which increased with density. We also note that the suppressed metabolic rates did not induce any type of encystation or other state change, and that cells were active and still dividing at the lowest measured metabolic rates.

Division rates were positively correlated with metabolic rate (Figure 3), confirming our hypothesis that demographic parameters are linked to metabolic rate. The relationship was linear, which is consistent with Fenchel and Finlay's (1983) observation that production is linearly related to metabolic rate in protists. As expected, as metabolism fuels production (Brown and Sibly, 2006), individuals had the highest metabolic rates and highest division rates at low densities. Our estimated death rate function indicated that deaths were negligible until metabolic rates were highly suppressed (Figure 3). Intraspecific competition for food was apparently severe enough at high densities to cause individual cells to starve, as this seems to be the primary mode of death in this population. However, it is possible that some cells were cannibalized. Cannibalism has been reported for other species of Tetrahymena (Lynn 2008), but we have no direct evidence of this in our experimental populations, such as the presence of macrostome forms or actual observations of cannibalism under a microscope. Interestingly, this type of 'prey-switching' would be one way for organisms to avoid the metabolic suppression caused by increasing density, and would generally favor the diversification of consumeresource interactions in a population.

Thus, the decline in PGR with density may arise because of the negative effect of density on $\vec{B}_{\mathrm{pc}}$ and the subsequent effect of $\bar{B}_{\mathrm{pc}}$ on birth and death rates. 
The variation in the shape of the relationship between PGR and density (described in Sibly et al., 2005), therefore, depends on both the value of $\alpha$ and exactly how individual demographic rates (births and deaths) map onto $\bar{B}_{\mathrm{pc}}$. Furthermore, because closed populations change according to $\frac{d n}{d t}=(b-d) N$, we can substitute known or estimated birth and death rate functions in terms of metabolic rate, $b\left(\bar{B}_{\mathrm{pc}}\right)$ and $d\left(\bar{B}_{\mathrm{pc}}\right)$, to write energetically explicit models of population dynamics, which could generate new insights about the population behavior. For example, in our populations of T. pyriformis, the deceleration in growth (sigmoid growth pattern) was due to gradually slowing birth rates as metabolic rate rapidly declined. The approximate steady-state density was achieved only when death rates increased sharply at the lowest metabolic rates.

The value of our approach to studying population regulation as a two-stage energetic process is not merely to suggest where the energetic mechanisms of regulation lie, but to create a structure that allows us to explain a variety of population-level phenomenon. Many classic problems in population ecology, such as oscillating numbers, predator-prey cycles or the appearance of algal blooms, may all benefit by evaluating the density dependence of metabolic rate and investigating how this phenomenon generates particular demographic patterns that drive population behavior. Additional phenomena, such as the temperature-size rule (Atkinson et al., 2003), can be evaluated for their population effects by incorporating size and temperature effects on metabolic rate, and tracking the response through the density dependence of metabolic rate and the subsequent birth and death rates. Thus, our results illustrate one way in which the widespread phenomenon of density-dependent PGR may have a physiological foundation, and they provide a new approach for investigating more complex population phenomenon that often proves difficult to understand.

\section{Acknowledgements}

We appreciate the input of J Brown, O Burger, L Marschall, $\mathrm{T}$ Meehan, R Sibly, $\mathrm{P}$ Turchin and several anonymous reviewers. The programming assistance from $\mathrm{E}$ Toolson was indispensable. We also greatly appreciate the laboratory assistance of $U$ Bergthorsson and his laboratory members. Funds for this work came from UNM GRAC, SRAC, and a Grove Summer Scholarship to JPD. Funding was also provided by UNM research allocation committee Grant \#05-L-07 to DTH.

\section{References}

Atkinson D, Ciotti BJ, Montagnes DJS. (2003). Protists decrease in size linearly with temperature: ca $2.5 \%$ ${ }^{\circ} \mathrm{C}^{-1}$. Proc Roy Soc Lond B 270: 2605-2611.

Berryman AA, Arce ML, Hawkins BA. (2002). Population regulation, emergent properties, and a requiem for density dependence. Oikos 99: 600-606.
Brown JH, Gillooly JF, Allen AP, Savage VM, West GB. (2004). Toward a metabolic theory of ecology. Ecology 85: 1771-1789.

Brown JH, Sibly RM. (2006). Life-history evolution under a production constraint. Proc Natl Acad Sci USA 103: 17595-17599.

Bulmer MG. (1975). The statistical analysis of density dependence. Biom 31: 901-911.

Cameron IL, Jeter JR. (1970). Synchronization of the cell cycle of Tetrahymena by starvation and refeeding. J Protoz 17: 429-433.

DeLong JP, Hanson DT. (2009). Density-dependent individual and population-level metabolic rates in a suite of single-celled eukaryotes. Open Biol J 2: 32-37.

Fenchel T, Finlay BJ. (1983). Respiration rates in heterotrophic, free-living protozoa. Microb Ecol 9: 99-122.

Forrester GE, Evans B, Steele MA, Vance RR. (2006). Assessing the magnitude of intra- and interspecific competition in two coral reef fishes. Oecologia 148: 632-640.

Gause GF. (1934). The Struggle For Existence. Williams and Wilkins: Baltimore, (Reprinted 1964 by Hafner).

Gillooly JF, Brown JH, West GB, Savage VM, Charnov EL. (2001). Effects of size and temperature on metabolic rate. Science 293: 2248-2251.

Hixon MA, Carr MH. (1997). Synergistic predation, density dependence, and population regulation in marine fish. Science 277: 946-949.

Lambers JHR, Clark JS, Beckage B. (2002). Densitydependent mortality and the latitudinal gradient in species diversity. Nature 417: 732-735.

Lewis S, Sherratt TN, Hamer KC, Wanless S. (2001). Evidence of intra-specific competition for food in a pelagic seabird. Nature 412: 816-819.

Lynn DH. (2008). The Ciliated Protozoa: Characterization, Classification, and Guide to the Literature, 3rd edn. Springer: Berlin, Germany.

Nässberger L, Monti M. (1984). Assessment of overall metabolism in Amoeba proteus measured by a microcalorimetric method. Protoplasma 123: 135-139.

Ohman MD, Hirche H-J. (2001). Density-dependent mortality in an oceanic copepod population. Nature 412: 638-641.

Pace DM, Kimura TE. (1944). The effect of temperature on respiration in Paramecium aurelia and Paramecium caudatum. J Cell Comp Phys 24: 173-183.

Pearl R. (1927). The growth of populations. Q Rev Biol 2: 532-548.

Schmoker C, Hernandez-Leon S. (2003). The effect of food on the respiration rates of Daphnia magna using a flow-through system. Scientia Marina 67: 361-365.

Secor SM, Diamond J. (1998). A vertebrate model of extreme physiological regulation. Nature 395: 659-662.

Sibly R, Barker D, Denham MC, Hone J, Pagel M. (2005). On the regulation of populations of mammals, birds, fish, and insects. Science 309: 607-610.

Tonn WN, Holopainen IJ, Paszkowski CA. (1994). Densitydependent effects and the regulation of Crucian carp populations in single-species ponds. Ecol 75: 824-834.

Turchin P. (1999). Population regulation: a synthetic view. Oikos 84: 153-159.

van Aarde R, Whyte I, Pimm S. (1999). Culling and the dynamics of the Kruger National Park African elephant population. Anim Cons 2: 287-294.

Woiwod IP, Hanski I. (1992). Patterns of density dependence in moths and aphids. $J$ Anim Ecol 61: 619-629. 